\title{
On the Thermodynamic Properties of the Spinless Duffin-Kemmer-Petiau Oscillator in Noncommutative Plane
}

\author{
Zhi Wang, Zheng-Wen Long, Chao-Yun Long, and Wei Zhang \\ Department of Physics, Guizhou University, Guiyang 550025, China \\ Correspondence should be addressed to Zheng-Wen Long; phy.zhenwen@gmail.com
}

Received 15 July 2015; Revised 30 September 2015; Accepted 4 October 2015

Academic Editor: Elias C. Vagenas

Copyright ( 2015 Zhi Wang et al. This is an open access article distributed under the Creative Commons Attribution License, which permits unrestricted use, distribution, and reproduction in any medium, provided the original work is properly cited. The publication of this article was funded by SCOAP $^{3}$.

The Duffin-Kemmer-Petiau oscillator for spin 0 particle in noncommutative plane is analyzed and the energy eigenvalue of the system is obtained by employing the functional analysis method. Furthermore, the thermodynamic properties of the noncommutative DKP oscillator are investigated via numerical method and the influence of noncommutative space on thermodynamic functions is also discussed. We show that the energy spectrum and corresponding thermodynamic functions of the considered physical systems depend explicitly on the noncommutative parameter $\theta$ which characterizes the noncommutativity of the space.

\section{Introduction}

The Duffin-Kemmer-Petiau (DKP) equation introduced by Kemmer et al. [1-3] in the 1930s is a relativistic wave equation which describes spin 0 and spin 1 bosons in the description of the standard model. The DKP equation for spin 0 hadrons with nuclei is closely linked to the conventional secondorder Klein-Gordon (KG) equation, which has more complex algebraic structure than the latter [4], and the DKP equation enjoys a richness of couplings not capable of being expressed in the Klein-Gordon and Proca equations [5, 6]. In addition, several situations researched in past years involving hadronic process and breaking of symmetries show that in some situations the DKP equation and KG equation can give some different results. For these reasons, there has been a growing interest in studying the DKP equation [7-13].

On the other hand, the noncommutative (NC) geometry has been put forward in both quantum mechanics and field theory in past years, which is growing rapidly. The coordinate noncommutativity was first introduced by Snyder [14] as a regularized mechanism to improve the problem of infinite self-energy in quantum field theory. But with the development of renormalization theory, this concept became not popular until Connes [15] investigated Yang-Mills theories in noncommutative (NC) space. In recent years, there has been a renewed interest in NC geometry because of the discovery in string theory and matrix model of M-theory [16]. When studying NC space, one considers the coordinate to be noncommutative, where the coordinate operators satisfy the commutation relation $\left[\widehat{x}_{i}, \widehat{x}_{i}\right]=i \theta_{i j}$, with $\theta_{i j}$ being a constant antisymmetric matrix, which represent the noncommutative property of NC space, and play an analogous role with $\hbar$ in the usual quantum mechanics. Recently, various aspects of both NC classical [17] and quantum [18] mechanics have been investigated by large number of papers, devoted to exploring the role of NC parameter in the physical observables. For example, classical Newton mechanics in the noncommutative space was studied in $[19,20]$, a particle confined by a quadratic potential in the generalized noncommutative plane was investigated from both the classical and the quantum aspects in [21], the noncommutative harmonic oscillators were discussed in detail in [22-24], and [25] studied the Aharonov-Bohm effect in a class of noncommutative theories.

The DKP equation in the presence of the Dirac oscillator interaction called DKP oscillator for spin zero and spin one particles has also been investigated in the NC formalism in publications [26-28], with the purpose of obtaining the energy spectrum and studying the noncommutative effect on relativistic quantum mechanics. In addition, the studies of thermal properties of the quantum oscillator have been 
carried out in both commutative and NC space by several authors these years [29-34]. By employing the numerical method based on the Euler-MacLaurin formula, one can calculate the associated partition function and obtain the thermodynamic functions, such as the free energy, mean energy, heat capacity, and entropy. In spite of several papers that have been published concerning the thermodynamics properties of quantum oscillator, as far as we know they have not reported the case of DKP oscillator for spin 0 particle in NC space. It will be interesting to study thermodynamic properties of the spinless DKP oscillator in NC formalism.

This work is organized as follows: In Section 2 we briefly review the NC quantum mechanics, where the NC space is introduced. Subsequently, we analyze the solutions of the DKP oscillator for spin 0 particle in NC plane and obtain the corresponding energy spectrum. The thermodynamic properties of the NC DKP oscillator are investigated by employing the Euler-MacLaurin method in Section 3. To have an intuitive understanding for the statistical properties of the physical system, we depict the numerical results of the thermodynamic functions with several figures and discuss the effect of the NC parameter on thermal properties in Section 4. In addition, we also discuss the case of low temperature limit based on the Hurwitz zeta function formula. Finally, the work is summarized in last section.

\section{The DKP Oscillator for Spin 0 Particle in NC Plane}

2.1. Review of the NC Formalism. In NC space, the canonical variables satisfy the following commutation relations:

$$
\begin{aligned}
& {\left[\widehat{x}_{i}, \widehat{x}_{j}\right]=i \theta_{i j},} \\
& {\left[\widehat{p}_{i}, \widehat{p}_{j}\right]=0,} \\
& {\left[\widehat{x}_{i}, \widehat{p}_{j}\right]=i \hbar \delta_{i j},}
\end{aligned}
$$

$$
(i, j=1,2),
$$

with $\theta_{i j}=\varepsilon_{i j} \theta$ being the antisymmetric NC parameter, representing the noncommutativity of the space, and $\widehat{x}_{i}, \widehat{p}_{i}$ are the coordinate and momentum operators in the NC space.

One way to deal with the problem of noncommutative space is via the star product or Moyal-Weyl product on the commutative space functions:

$$
(f * g)(x)=\left.\exp \left(\frac{i}{2} \theta_{i j} \partial_{i}^{x} \partial_{j}^{y}\right) f(x) g(y)\right|_{y \rightarrow x},
$$

where $f(x)$ and $g(y)$ are arbitrary infinitely differentiable functions.

Then the time-independent Schrödinger equation in the NC space can be written in the form

$$
H(x, p) * \psi=E \psi
$$

The Moyal-Weyl product may be replaced by a Bopp shift of the form

$$
x_{i} \longrightarrow x_{i}-\frac{1}{2 \hbar} \theta_{i j} p_{j}, \quad p_{i} \longrightarrow p_{i}
$$

where $x_{i}$ and $p_{i}$ are the position and momentum operators in the usual quantum mechanics which meet the canonical Heisenberg commutation relations.

Then, via above Bopp shift, the Schrödinger equation can be written as

$$
H\left(x_{i} \longrightarrow x_{i}-\frac{1}{2 \hbar} \theta_{i j} p_{j}, p_{i}\right) \psi=E \psi .
$$

Therefore, (5) is actually defined on the commutative space, and the NC effect may be calculated from the term that contains $\theta$.

2.2. The Solution of the DKP Oscillator for Spin 0 Particle in NC Plane. The relativistic DKP equation for a free boson of mass $m$ is given by $[35,36]$

$$
\left[c \vec{\beta} \cdot \vec{P}+m c^{2}\right] \psi=i \hbar \beta^{0} \frac{\partial}{\partial t} \psi,
$$

with $\vec{\beta}=\left(\beta^{1}, \beta^{2}, \beta^{3}\right)$ and $\beta^{0}$ being the DKP matrices which satisfy the following algebra relation:

$$
\beta^{\mu} \beta^{v} \beta^{\lambda}+\beta^{\lambda} \beta^{v} \beta^{\mu}=g^{\mu \nu} \beta^{\lambda}+g^{\lambda v} \beta^{\mu},
$$

where $g^{\mu \nu}=\operatorname{diag}(1,-1,-1,-1)$ is the metric tensor in Minkowski space. For spin 0 particle, $\beta^{\mu}$ are $5 \times 5$ matrices written as

$$
\begin{aligned}
& \beta^{0}=\left[\begin{array}{ll}
I_{0} & \overline{0} \\
\overline{0}^{T} & 0
\end{array}\right], \\
& \beta^{i}=\left[\begin{array}{cc}
\tilde{0} & \rho_{\iota} \\
-\left(\rho_{\iota}\right)^{T} & 0
\end{array}\right], \quad \iota=1,2,3,
\end{aligned}
$$

with $0, \overline{0}$, and $\widetilde{0}$ being $3 \times 3,2 \times 3$, and $2 \times 2$ zero matrices, respectively. Other matrices in (8) are given as follows:

$$
\begin{aligned}
& I_{0}=\left[\begin{array}{ll}
0 & 1 \\
1 & 0
\end{array}\right], \\
& \rho_{1}=\left[\begin{array}{ccc}
-1 & 0 & 0 \\
0 & 0 & 0
\end{array}\right], \\
& \rho_{2}=\left[\begin{array}{ccc}
0 & -1 & 0 \\
0 & 0 & 0
\end{array}\right], \\
& \rho_{3}=\left[\begin{array}{lll}
0 & 0 & -1 \\
0 & 0 & 0
\end{array}\right] .
\end{aligned}
$$

Introducing the nonminimal substitution $\left(\vec{P} \rightarrow \vec{P}-i m \omega \eta^{0} \vec{r}\right)$ in (6), we get the DKP oscillator as

$$
\left[c \vec{\beta} \cdot\left(\vec{P}-i m \omega \eta^{0} \vec{r}\right)+m c^{2}\right] \psi=i \hbar \beta^{0} \frac{\partial}{\partial t} \psi
$$

with $\omega$ being the oscillator frequency and $\eta^{0}=2\left(\beta^{0}\right)^{2}-1$. 
It is easy to get the 2D DKP oscillator from the above equation:

$$
\begin{aligned}
& {\left[c \beta^{1}\left(p_{x}-i m \omega \eta^{0} x\right)+c \beta^{2}\left(p_{y}-i m \omega \eta^{0} y\right)+m c^{2}\right] \psi} \\
& =\beta^{0} E \psi .
\end{aligned}
$$

Considering the NC formalism and via the Bopp shift (4), the DKP oscillator of (11) can be written as

$$
\begin{aligned}
& {\left[c \beta^{1}\left(p_{x}-i m \omega \eta^{0}\left(x-\frac{\theta}{2 \hbar} p_{y}\right)\right)\right.} \\
& \left.\quad+c \beta^{2}\left(p_{y}-i m \omega \eta^{0}\left(y+\frac{\theta}{2 \hbar} p_{x}\right)\right)+m c^{2}\right] \psi \\
& \quad=\beta^{0} E \psi .
\end{aligned}
$$

For a boson of spin 0 , the spinor $\psi$ is a vector with five components $[9,10]$, which has the form $\psi=\left(\psi_{1}, \psi_{2}, i \phi_{1}, i \phi_{2}, i \phi_{3}\right)^{T}$.

Substituting $\psi$ into (12), one can obtain the following five coupled equations:

$$
\begin{aligned}
& -m c^{2} \psi_{1}+E \psi_{2}+i c\left[p_{x}+i m \omega\left(x-\frac{\theta}{2 \hbar} p_{y}\right)\right] \phi_{3} \\
& +i c\left[p_{y}+i m \omega\left(y+\frac{\theta}{2 \hbar} p_{x}\right)\right] \phi_{4}=0, \\
& E \psi_{1}-m c^{2} \psi_{2}=0 \\
& c\left[p_{x}-i m \omega\left(x-\frac{\theta}{2 \hbar} p_{y}\right)\right] \psi_{1}+i m c^{2} \phi_{3}=0, \\
& c\left[p_{y}-i m \omega\left(y+\frac{\theta}{2 \hbar} p_{x}\right)\right] \psi_{1}+i m c^{2} \phi_{4}=0, \\
& i m c^{2} \phi_{5}=0 .
\end{aligned}
$$

Combination of (13) gives

$$
\begin{gathered}
{\left[\left(\frac{1}{2 m}+\frac{m \omega^{2} \theta^{2}}{8 \hbar^{2}}\right)\left(p_{x}^{2}+p_{y}^{2}\right)+\frac{m \omega^{2}}{2}\left(x^{2}+y^{2}\right)\right.} \\
\left.-\frac{m \omega^{2} \theta}{2 \hbar} L_{z}\right] \psi_{1}=\frac{E^{2}-m^{2} c^{4}+2 m \omega \hbar c^{2}}{2 m c^{2}} \psi_{1} .
\end{gathered}
$$

Using polar coordinates, the component $\psi_{1}$ can be written as

$$
\psi_{1}(\rho, \varphi)=R(\rho) \exp \left(i m_{l} \varphi\right), \quad m_{l}=0, \pm 1, \pm 2, \ldots,
$$

and substituting it into (14), we can obtain the radial equation

$$
\left[\frac{d^{2}}{d \rho^{2}}+\frac{1}{\rho} \frac{d}{d \rho}-\frac{m_{l}^{2}}{\rho^{2}}-\beta \rho^{2}+\gamma\right] \psi=0,
$$

with

$$
\begin{aligned}
& \beta=\frac{4 m^{2} \omega^{2}}{m^{2} \omega^{2} \theta^{2}+4 \hbar^{2}}, \\
& \gamma=\frac{E^{2}+m_{l} m^{2} c^{2} \omega^{2} \theta-m^{2} c^{4}+2 m \omega \hbar c^{2}}{\left(\hbar^{2}+(1 / 4) m^{2} \omega^{2} \theta^{2}\right) c^{2}} .
\end{aligned}
$$

Introducing a new variable $\xi=\sqrt{\beta} \rho^{2}$, one can get the following second-order differential equation:

$$
\frac{d^{2} R(\xi)}{d \xi^{2}}+\frac{1}{\xi} \frac{d R(\xi)}{d \xi}-\left(\frac{m_{l}^{2}}{4 \xi^{2}}+\frac{1}{4}-\frac{\gamma}{4 \sqrt{\beta} \xi}\right) R(\xi)=0 .
$$

The equation can be analyzed asymptotically at the critical points $\xi \rightarrow 0$ and $\xi \rightarrow \infty$, allowing us to construct an equation whose form is the same as the confluent hypergeometric equation. This analysis shows that it is reasonable to write $R(\xi)$ as

$$
R(\xi)=\xi^{\left|m_{l}\right| / 2} e^{-\xi / 2} F(\xi) .
$$

Substituting the above function into (18), one obtains

$$
\begin{aligned}
\xi F^{\prime \prime}(\xi) & +\left(\left|m_{l}\right|+1-\xi\right) F^{\prime}(\xi) \\
& -\left(\frac{\left|m_{l}\right|}{2}+\frac{1}{2}-\frac{\gamma}{4 \sqrt{\beta}}\right) F(\xi)=0 .
\end{aligned}
$$

Obviously, this is confluent hypergeometric equation whose solution is the confluent hypergeometric function

$$
F(\xi)={ }_{1} F_{1}\left(\frac{\left|m_{l}\right|}{2}+\frac{1}{2}-\frac{\gamma}{4 \sqrt{\beta}},\left|m_{l}\right|+1 ; \xi\right) .
$$

To obtain the solution of (20), the polynomial series should be convergent. It requires the independent term to be negative integer [37]:

$$
\frac{\left|m_{l}\right|}{2}+\frac{1}{2}-\frac{\gamma}{4 \sqrt{\beta}}=-n
$$

Through a simple calculation we obtain the energy of the system as follows:

$$
\begin{aligned}
E^{2}= & 2 \hbar \omega m c^{2} \sqrt{1+\frac{m^{2} \omega^{2} \theta^{2}}{4 \hbar^{2}}}\left(2 n+\left|m_{l}\right|+1\right) \\
& -m_{l} m^{2} c^{2} \omega^{2} \theta+m^{2} c^{4}-2 \hbar \omega m c^{2} .
\end{aligned}
$$

The eigenvalues of considered physical system depend distinctly on the NC parameter $\theta$ which characterizes the noncommutativity of the space. We find that the energy spectrum is exact and not degenerate due to the NC effect.

Then $\psi_{1}(\rho, \varphi)$ can be written in the form of the confluent hypergeometric function as

$$
\begin{aligned}
& \psi_{1}(\rho, \varphi) \\
& =N e^{i m_{l} \varphi} \rho^{\left|m_{l}\right|} e^{-(\sqrt{\beta} / 2) \rho^{2}}{ }_{1} F_{1}\left(-n,\left|m_{l}\right|+1, \sqrt{\beta} \rho^{2}\right),
\end{aligned}
$$

where $\beta$ has already been given above, and through straightforward calculation, the expression of other components of the spinor $\psi$ and the final form can be obtained easily. 


\section{Thermodynamic Properties of the DKP Oscillator for Spin 0 Particle in NC Plane}

In this section, we study the thermodynamic properties of above DKP oscillator. The energy eigenvalues of the DKP oscillator in NC space are

$$
E_{n}= \pm m c^{2} \sqrt{\alpha n+\lambda}, \quad n=0,1,2, \ldots,
$$

with

$$
\begin{aligned}
\alpha= & \frac{4 \hbar \omega}{m c^{2}} \sqrt{1+\frac{m^{2} \omega^{2} \theta^{2}}{4 \hbar^{2}}}, \\
\lambda= & \frac{2 \hbar \omega}{m c^{2}} \sqrt{1+\frac{m^{2} \omega^{2} \theta^{2}}{4 \hbar^{2}}}\left(\left|m_{l}\right|+1\right)-\frac{m_{l} \omega^{2} \theta}{c^{2}}-\frac{2 \hbar \omega}{m c^{2}} \\
& +1 .
\end{aligned}
$$

In Figure 1, we plot the energy spectra $E$ versus quantum number $n$ for different values of the NC parameter $\theta^{\prime}$. Positive- and negative-energy levels correspond to the case of particle and antiparticle, respectively. We see that the energy $E$ increases monotonically with quantum number $n$ and the tendency of the spectrum can be observed for large quantum numbers. The effect of the NC parameter on the energy levels is observable, where $\theta^{\prime}=0$ corresponding to the case of commutative space.

Because all thermodynamic quantities can be obtained from the partition function $Z$, we first calculate the partition function of the system which is defined at temperature $T$ as

$$
Z(V, T)=\sum_{n=0}^{\infty} e^{-\beta\left(E_{n}-E_{0}\right)},
$$

where $\beta=1 / k_{B} T, k_{B}$ is the Boltzmann constant, and $E_{0}$ is ground state energy. For $N$-body systems with no interaction inside, the corresponding partition function of the DKP oscillator should be $(Z)^{N}$. Here for simplicity we just focus on the case of $N=1$.

Then the thermodynamic properties of the physical system, such as free energy, mean energy, specific heat, and entropy, can be calculated from the following expressions [31]:

$$
\begin{aligned}
& F=-\frac{1}{\beta} \ln (Z), \\
& U=-\frac{\partial \ln (Z)}{\partial \beta}, \\
& C=\frac{\partial U}{\partial T}=-k_{B} \beta^{2} \frac{\partial U}{\partial \beta}, \\
& S=-\frac{\partial F}{\partial T}=k_{B} \beta^{2} \frac{\partial F}{\partial \beta} .
\end{aligned}
$$

To perform our analysis on the thermodynamics of the DKP oscillator, we will restrict ourselves to stationary states of positive energy. The reasons are as follows [31, 38, 39].

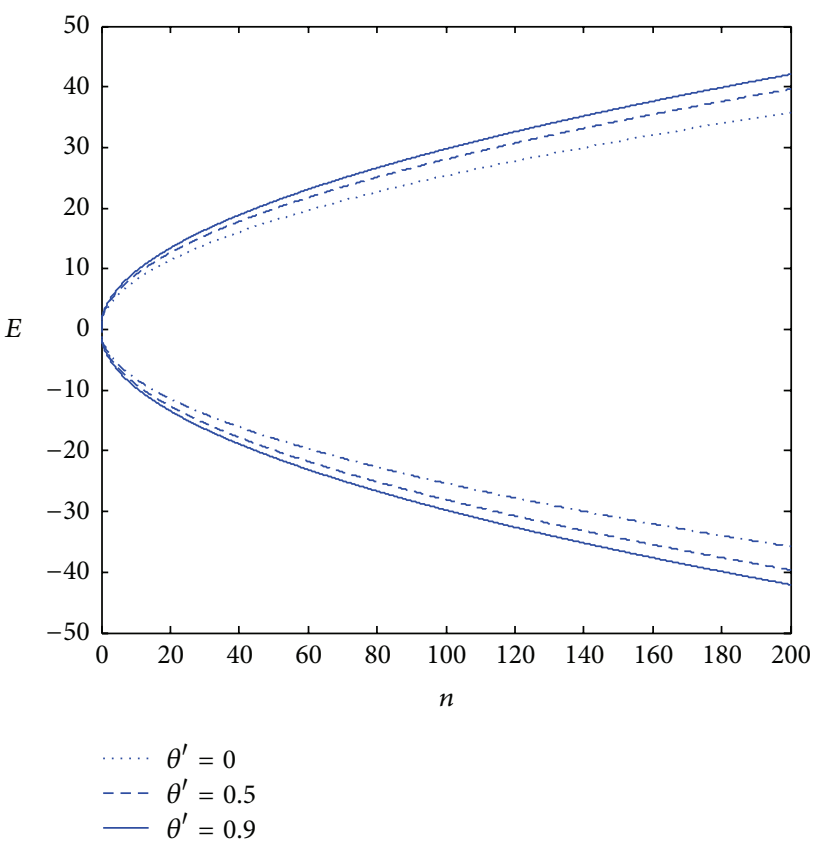

FIgURE 1: The profile of the energy spectra $E$ of the DKP oscillator on NC plane versus quantum number $n$ for different values of the NC parameter $\theta^{\prime}$

First, according to the study in [40, 41], the DKP equation possesses an exact Foldy-Wouthuysen transformation (FWT): thus, the positive- and negative-energy levels never mix. Second, although bosons can occupy the same level, which are different from the fermions controlled by the Pauli principle. We can still extend the method of filling the Dirac negative energy sea for the case of fermions to the one of bosons $[42,43]$ : as mentioned by the authors, the states of bosons are divided into two sectors: the usual positive sector consists of states with positive number of bosons, and the negative sector consists of states with negative number of bosons. Once a state enters the negative sector, it cannot return to the positive sector via an ordinary interaction due to the presence of a barrier.

In addition, for the negative-energy states, the partition function $Z$ of the DKP oscillator at finite temperature $T$ has the form

$$
Z=\sum_{n=0}^{\infty} e^{\beta m c^{2}(\sqrt{\alpha n+\lambda}-\sqrt{\lambda})}=e^{-\beta m c^{2} \sqrt{\lambda}} \sum_{n=0}^{\infty} e^{\beta m c^{2} \sqrt{\alpha n+\lambda}} .
$$

We apply the integral test to analyze the convergence of the above partition function. From (29), we can see that the function $f(x)=e^{\beta m c^{2} \sqrt{\alpha x+\lambda}}$ is an increasing positive function, and the integral

$$
\int_{0}^{\infty} f(x) d x=\int_{0}^{\infty} e^{\beta m c^{2} \sqrt{\alpha x+\lambda}} d x
$$

is not convergent. This means that, according to the criterion of the integral test, the numerical partition function $Z$ does not converge, while for the positive-energy states, it is obvious 
that the corresponding numerical partition function $Z$ is convergent.

According to the analysis above, we can assume that only particles with positive energy are available in order to set up a thermodynamic ensemble.

Now let us go on the main object of our interest, substituting (25) into (27), we obtain the partition function of the DKP oscillator in NC plane as

$$
Z=\sum_{n=0}^{\infty} e^{-\beta m c^{2}(\sqrt{\alpha n+\lambda}-\sqrt{\lambda})}=e^{\beta m c^{2} \sqrt{\lambda}} \sum_{n=0}^{\infty} e^{-\beta m c^{2} \sqrt{\alpha n+\lambda}}
$$

To calculate expression (31), we employ the Euler-MacLaurin formula

$$
\begin{aligned}
\sum_{n=0}^{\infty} f(n)= & \frac{1}{2} f(0)+\int_{0}^{\infty} f(x) d x \\
& -\sum_{p=1}^{\infty} \frac{1}{(2 p) !} B_{2 p} f^{(2 p-1)}(0),
\end{aligned}
$$

with $B_{2 p}$ being the Bernoulli numbers and

$$
f(x)=e^{-\beta m c^{2} \sqrt{\alpha x+\lambda}} .
$$

Then we have

$$
\begin{aligned}
& \int_{0}^{\infty} f(x) d x=\int_{0}^{\infty} e^{-\beta m c^{2} \sqrt{\alpha x+\lambda}} d x \\
& =\frac{2}{\alpha \beta^{2} m^{2} c^{4}}\left(\beta m c^{2} \sqrt{\lambda}+1\right) e^{-\beta m c^{2} \sqrt{\lambda}}, \\
& f^{(1)}(0)=-\frac{\alpha \beta m c^{2}}{2 \sqrt{\lambda}} e^{-\beta m c^{2} \sqrt{\lambda}}, \\
& f^{(3)}(0)=-\frac{\alpha^{3} \beta m c^{2}}{8}\left[3 \lambda^{-5 / 2}+3 \beta m c^{2} \lambda^{-2}\right. \\
& \left.+\beta^{2}\left(m c^{2}\right)^{2} \lambda^{-3 / 2}\right] e^{-\beta m c^{2} \sqrt{\lambda}} .
\end{aligned}
$$

Up to $B_{4}$, with $B_{2}=1 / 6, B_{4}=-1 / 30$, and combining with (34), we obtain the partition function at finite temperature $T$ as

$$
\begin{aligned}
Z= & \frac{1}{2}+\frac{2 \sqrt{\lambda_{0}}}{\alpha_{0}} \tau+\frac{2}{\alpha_{0}} \tau^{2} \\
& +\left(\frac{\alpha_{0}}{24 \sqrt{\lambda_{0}}}-\frac{\alpha_{0}^{3}}{1920 \sqrt{\lambda_{0}^{5}}}\right) \frac{1}{\tau}-\frac{\alpha_{0}^{3}}{1920 \lambda_{0}^{2}} \frac{1}{\tau^{2}} \\
& -\frac{\alpha_{0}^{3}}{5760 \sqrt{\lambda_{0}^{3}}} \frac{1}{\tau^{3}},
\end{aligned}
$$

with

$$
\begin{aligned}
\alpha_{0} & =4 \xi \sqrt{1+\theta^{\prime}}, \\
\lambda_{0} & =2 \xi \sqrt{1+\theta^{\prime}}-2 \xi+1, \\
\tau & =\frac{1}{\beta m c^{2}}=\frac{k_{B} T}{m c^{2}}, \\
\xi & =\frac{\hbar \omega}{m c^{2}}, \\
\theta^{\prime} & =\frac{m^{2} \omega^{2} \theta^{2}}{4 \hbar^{2}},
\end{aligned}
$$

where the dimensionless variable $\tau$ is proportional to temperature $T$ and $\theta^{\prime}$ characterizes the noncommutativity of the space. From (35), we predict that the thermodynamic functions will be very complicated. To have an intuitive understanding for thermodynamic properties of the spinless DKP oscillator on NC plane, in what follows, we briefly depict our numerical results on the evaluation of the thermodynamic functions, that is, free energy, mean energy, specific heat, and entropy, via the numerical partition function $Z$.

\section{Results and Discussions}

In this section, all profiles of the thermodynamic quantities as a function of dimensionless temperature variable $\tau$ for different values of the NC parameter $\theta^{\prime}$, that is, $\theta^{\prime}=0,0.5$, and 0.9 , are plotted in Figures 2-6. In these figures, the natural unit ( $\left.\hbar=c=k_{B}=1\right)$ is employed and we set $m=1$ and $\xi=1.5$.

From the result shown in Figure 2, we see that the partition function $Z$ increases monotonically with dimensionless variable $\tau$, and for a fixed value of $\tau$ the partition function decreases when NC parameter $\theta^{\prime}$ grows. It also shows that the partition function first has a slow-growth and then rapidly increases. The Helmholtz free energy $F$ is shown in Figure 3. We see that in all cases the profile of the curves decreases monotonically with the temperature and the free energy increase with NC parameter $\theta^{\prime}$ growing for a fixed value of $\tau$. In Figure 4, we plot the mean energy $U$ versus $\tau$ for different values of the NC parameter $\theta^{\prime}$, and it shows that all the curves have similar linear behavior and display very close profiles. To have better understanding of the NC effect on mean energy, we choose $\theta^{\prime}=0,0.5$, and 0.9 . And we also see that for a fixed $\tau$ the mean energy decreases when $\theta^{\prime}$ grows. The profile of heat capacity $C$ as a function of $\tau$ for different NC parameter $\theta^{\prime}$ values is depicted in Figure 5. It is observed that the heat capacity increases for increasing $\tau$ at first and then decreases with $\tau$ growing. There is an abrupt change around $\tau_{0}$ for each curve, and $\tau_{0}$ increases when the corresponding $\theta^{\prime}$ grows. Figure 6 displays the curves of the numerical entropy $S$ versus $\tau$ for different values of the NC parameter $\theta^{\prime}$. It shows that the tendency of entropy rapidly increases at first for increasing $\tau$ and then slowly grows for large $\tau$ values. For a fixed value of $\tau$, the entropy decreases when NC parameter $\theta^{\prime}$ grows. In addition, from the curves of the numerical mean energy, free energy, and entropy function, no abrupt changes around $\tau_{0}$ 


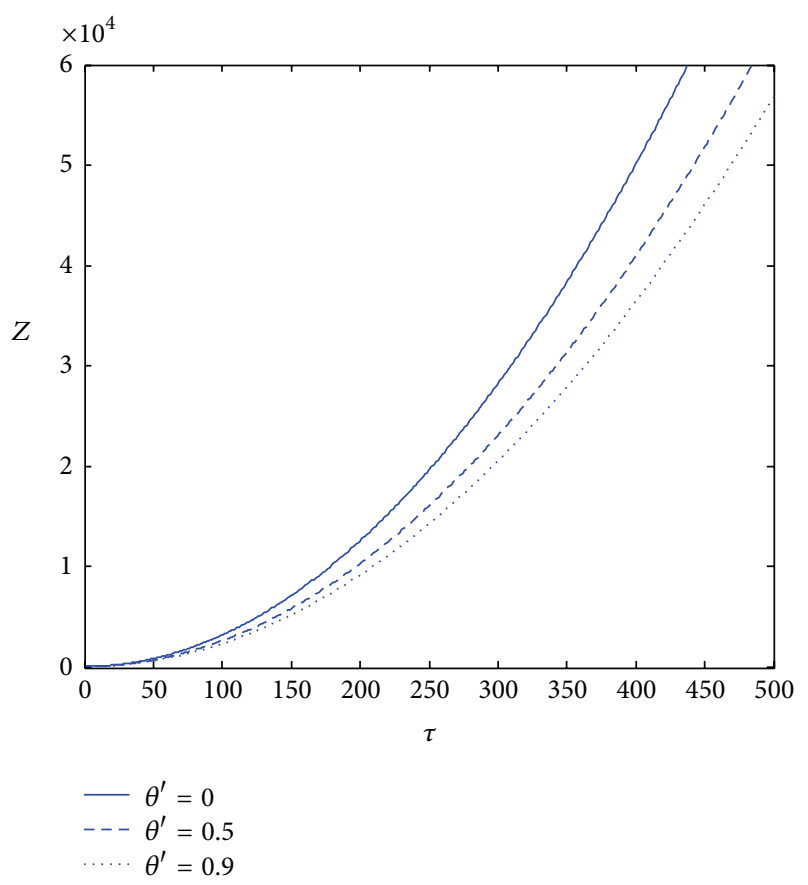

FIgURE 2: The partition function $Z$ of the DKP oscillator on NC plane as a function of $\tau$ for different values of the NC parameter $\theta^{\prime}$.

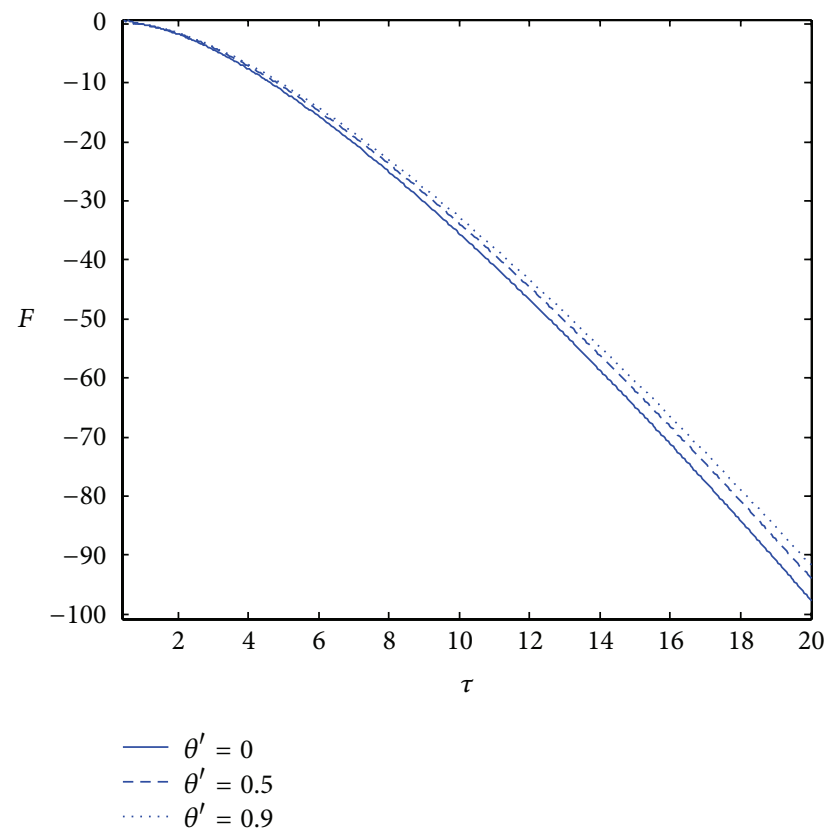

FIgURE 3: The free energy $F$ of the DKP oscillator in NC plane as a function of $\tau$ for different values of the NC parameter $\theta^{\prime}$.

have been identified, meaning that the curvature, observed in the specific heat curve, does not exhibit or indicate an existence of a phase transition around a finite temperature $\tau_{0}$.

Because the dimensionless variable $\tau$ is proportional to temperature $T$, we can get the characters of these thermodynamic functions at high temperature from the asymptotic behavior shown in the above figures. As is shown, the values

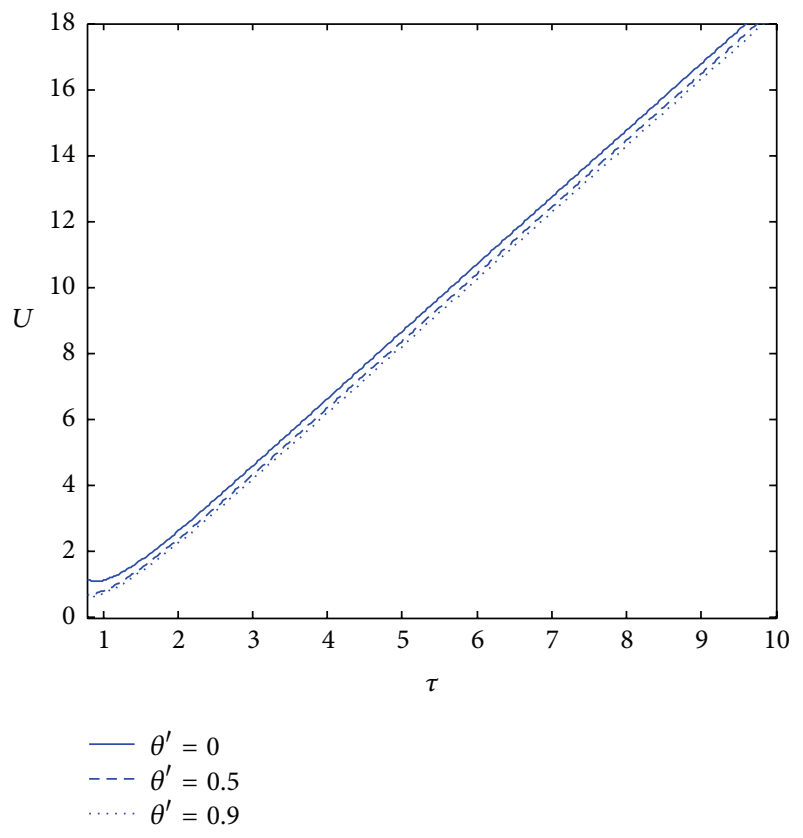

Figure 4: The mean energy $U$ of the DKP oscillator in NC plane as a function of $\tau$ for different values of the NC parameter $\theta^{\prime}$.

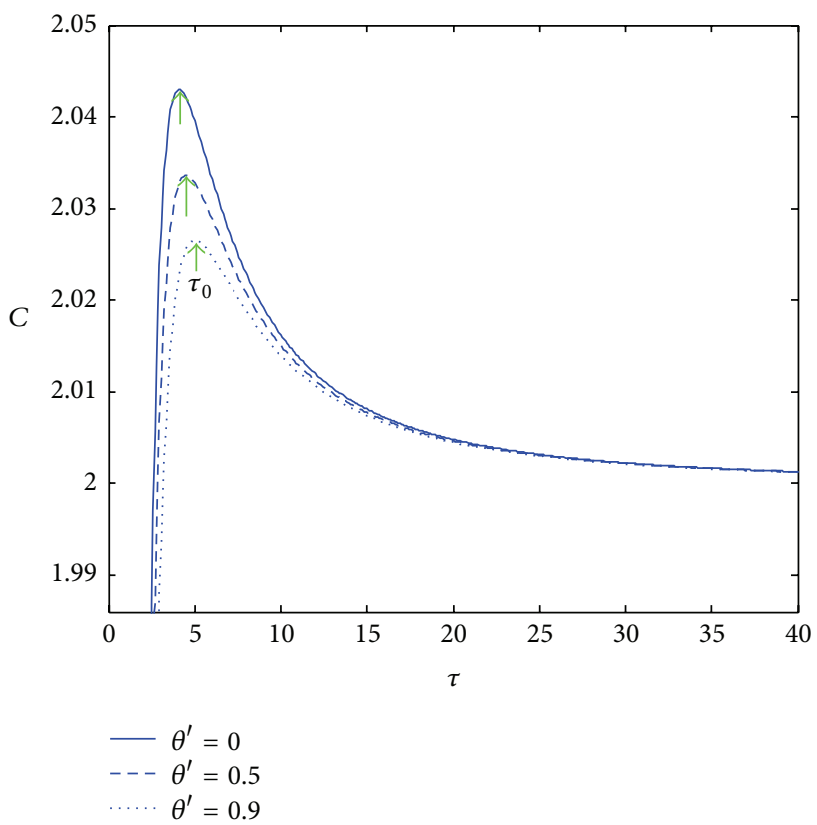

FIgUre 5: The heat capacity $C$ of the DKP oscillator in NC plane as a function of $\tau$ for different values of the NC parameter $\theta^{\prime}$.

of mean energy $U$ and entropy $S$ are large at high temperature, because these functions increase monotonically with dimensionless variable $\tau$. Contrarily, free energy $F$ will become very small at high temperature because it decreases monotonically with $\tau$ growing. The heat capacity $C$ will tend to a constant value at high temperature. The effect of the NC parameter $\theta^{\prime}$ on thermodynamic properties at high 


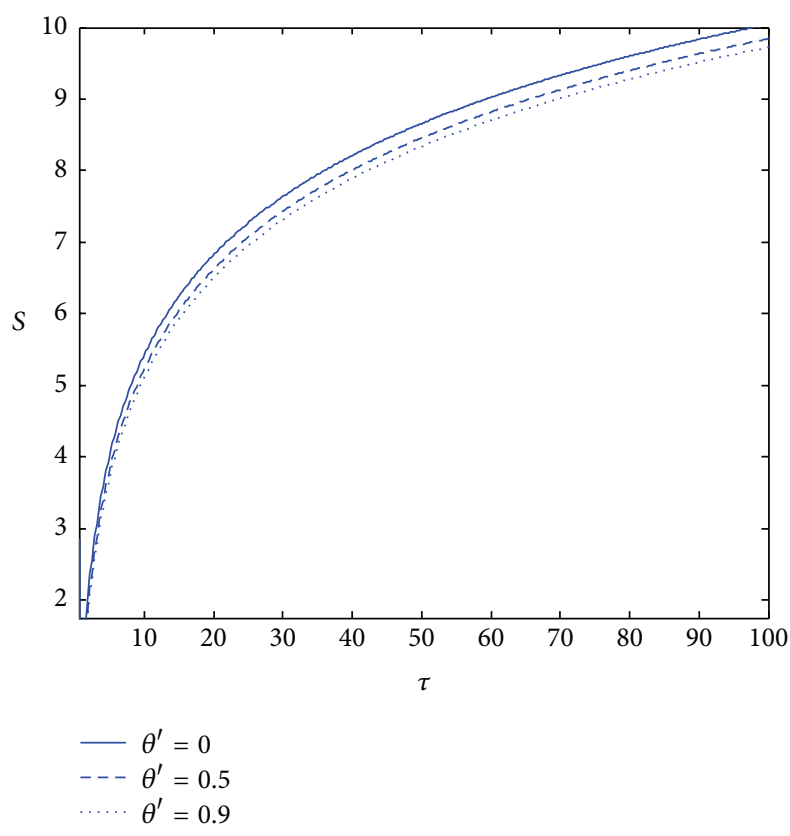

FIGURE 6: The entropy $S$ of the DKP oscillator in NC plane as a function of $\tau$ for different values of the NC parameter $\theta^{\prime}$.

temperature is observable. We see that the values of mean energy $U$ and entropy $S$ at high temperature case decrease with NC parameter $\theta^{\prime}$ growing, and in contrast the value of free energy $F$ increases when $\theta^{\prime}$ grows, while from Figure 5, we found that all the curves of heat capacity against different NC parameter $\theta^{\prime}$ at high temperature would tend to same value. It means that the effect of noncommutativity on the heat capacity can be negligible at high temperature.

Recently, Hassanabadi et al. have studied the spin 0 scalar bosons in presence of a uniform magnetic field by using the Klein-Gordon equation in NC space [33]. Similarly, with the numerical method based on the Euler-MacLaurin formula, the authors calculate the associated partition function and obtain the thermodynamic functions such as the free energy, mean energy, heat capacity, and entropy. Now we will compare our results with those obtained by them. For the free energy, mean energy, and entropy, it is easy to find that our result is consistent with theirs, and the curves have similar profiles, while the curve of heat capacity seems a little different: our result about the heat capacity will tend to a constant value $C_{0}$ at high temperature compared with the result shown in [33]. This divergence may be result of the different range of variable $\tau$.

In the above work, we have studied the thermodynamic properties of the NC DKP oscillator by employing the EulerMacLaurin approximation. According to the literature [38, 39], the formalism is only valid for high temperature because the partition function diverges in the low temperature limit $\tau \rightarrow 0$. In order to cover all range of temperature, one can use a method based on the Hurwitz zeta function which has been recently proposed by Boumali. In the following we will discuss the case of low temperature limit by using zeta function method.
Given the energy spectrum (25), one can define the partition via $[38,39]$

$$
Z=\sum_{n=0}^{\infty} e^{-\beta E_{n}}=\sum_{n=0}^{\infty} e^{-(\gamma / \tau) \sqrt{n+\kappa}},
$$

with the following substitutions:

$$
\begin{aligned}
& \gamma=\sqrt{\alpha_{0}}, \\
& \kappa=\frac{\lambda_{0}}{\alpha_{0}},
\end{aligned}
$$

where $\alpha_{0}, \lambda_{0}$, and $\tau$ are given in (36), using the formula [44]

$$
e^{-x}=\frac{1}{2 \pi i} \int_{c} d s x^{-s} \Gamma(s) .
$$

Then (38) is transformed into

$$
\begin{aligned}
\sum_{n=0}^{\infty} e^{-(\gamma / \tau) \sqrt{n+\kappa}} & =\frac{1}{2 \pi i} \int_{c} d s\left(\frac{\gamma}{\tau}\right)^{-s} \sum_{n}(n+\kappa)^{-s / 2} \Gamma(s) \\
& =\frac{1}{2 \pi i} \int_{c} d s\left(\frac{\gamma}{\tau}\right)^{-s} \zeta_{H}\left(\frac{s}{2}, \kappa\right) \Gamma(s),
\end{aligned}
$$

where $x=(\gamma / \tau) \sqrt{n+\kappa}$ and $\Gamma(s)$ and $\zeta_{H}(s / 2, \kappa)$ are the Euler and Hurwitz zeta function, respectively. Employing the residues theorem, for the two poles $s=0$ and $s=2$, the associated partition function is written in terms of the Hurwitz zeta function as follows:

$$
Z(\tau)=\left(\frac{\tau}{\gamma}\right)^{2}+\zeta_{H}(0, \kappa)
$$

Now, using the expression

$$
\zeta_{H}(0, \kappa)=\frac{1}{2}-\kappa,
$$

the final partition function becomes

$$
Z(\tau)=\frac{\tau^{2}}{\alpha_{0}}+\frac{1}{2}-\frac{\lambda_{0}}{\alpha_{0}}
$$

Then, all thermal quantities of the DKP oscillators, such as the free energy $F$, mean energy $U$, specific heat $C$, and entropy $S$, can be obtained by using the following expressions derived from (28) as

$$
\begin{aligned}
& F=-m c^{2} \tau \ln (Z), \\
& U=m c^{2} \tau^{2} \frac{\partial \ln (Z)}{\partial \tau}, \\
& S=k_{B} \ln (Z)+k_{B} \tau \frac{\partial \ln (Z)}{\partial \tau}, \\
& C=2 k_{B} \tau \frac{\partial \ln (Z)}{\partial \tau}+k_{B} \tau^{2} \frac{\partial^{2} \ln (Z)}{\partial \tau^{2}} .
\end{aligned}
$$

From expression (44), we see that the partition function is not divergent at the low temperature limit $\tau \rightarrow 0$; thus the result can be considered as an improvement of the result obtained by using the Euler-MacLaurin method. 


\section{Conclusions}

This paper was devoted to study of the thermodynamic properties of the DKP oscillator for spin 0 particle in noncommutative space. We first analyzed the DKP equation in the presence of the Dirac oscillator interaction in NC space and obtained energy spectrum as well as corresponding spinor $\psi$ of the system by employing the functional analysis method. Subsequently, we investigated the thermodynamic properties of the system by employing the Euler-MacLaurin method and depicted our numerical results on the evaluation of the thermodynamic functions through the associated partition function $Z$. And the effect of the NC parameter on thermodynamic properties was discussed. We show that the energy spectrum and corresponding thermodynamic functions depend explicitly on the NC parameter $\theta$ which characterizes the noncommutativity of the space. It is also observed that the influence of NC space on the thermodynamic properties is nonignorable although the NC parameter is small. In addition, we discuss the case of low temperature limit based on the Hurwitz zeta function formula, which can be considered as an improvement of the Euler-MacLaurin method. This work will allow us to further explore the role of NC space in the related physical concepts and deepen our knowledge on NC quantum mechanics.

\section{Conflict of Interests}

The authors declare that there is no conflict of interests regarding the publication of this paper.

\section{Acknowledgments}

The authors would like to express their gratitude to the referees for their valuable suggestions and critical comments. This work is supported by the National Natural Science Foundation of China (Grant nos. 10865003, 11465006, and 11565009).

\section{References}

[1] N. Kemmer, "Quantum theory of einstein-bose particles and nuclear interaction," Proceedings of the Royal Society of London Series A: Mathematical and Physical Sciences, vol. 166, no. 924, pp. 127-153, 1938.

[2] R. J. Duffin, "On the characteristic matrices of covariant systems," Physical Review, vol. 54, no. 12, p. 1114, 1938.

[3] G. Petiau, "University of Paris thesis," Académie Royale De Belgique. Classe Des Sciences. Mémoires. Collection, vol. 16, no. 2, p. 1, 1936.

[4] V. Y. Fainberg and B. M. Pimentel, "Duffin-Kemmer-Petiau and Klein-Gordon-Fock equations for electromagnetic, YangMills and external gravitational field interactions: proof of equivalence," Physics Letters A, vol. 271, no. 1-2, pp. 16-25, 2000.

[5] R. F. Guertin and T. L. Wilson, "Noncausal propagation in spin0 theories with external field interactions," Physical Review D, vol. 15, no. 6, pp. 1518-1531, 1977.

[6] B. Vijayalakshmi, M. Seetharaman, and P. M. Mathews, "Consistency of spin-1 theories in external electromagnetic fields," Journal of Physics A: Mathematical and General, vol. 12, no. 5, pp. 665-677, 1979.
[7] I. Boztosun, M. Karakoc, F. Yasuk, and A. Durmus, "Asymptotic iteration method solutions to the relativistic Duffin-KemmerPetiau equation," Journal of Mathematical Physics, vol. 47, no. 6, Article ID 062301, 2006.

[8] A. Boumali, "The eigensolutions of a two-dimensional Kemmer oscillator," Journal of Physics A: Mathematical and Theoretical, vol. 42, no. 23, Article ID 235301, 2009.

[9] H. Hassanabadi, B. H. Yazarloo, S. Zarrinkamar, and A. A. Rajabi, "Duffin-Kemmer-Petiau equation under a scalar Coulomb interaction," Physical Review C, vol. 84, no. 6, Article ID 064003, 2011.

[10] L. B. Castro and L. P. de Oliveira, "Remarks on the spin-one duffin-kemmer-petiau equation in the presence of nonminimal vector interactions in $(3+1)$ dimensions," Advances in High Energy Physics, vol. 2014, Article ID 784072, 8 pages, 2014.

[11] L. B. Castro and A. S. de Castro, "Spinless bosons embedded in a vector Duffin-Kemmer-Petiau oscillator," Physics Letters A, vol. 375, no. 27, pp. 2596-2600, 2011.

[12] L. B. Castro and A. S. de Castro, "Corroborating the equivalence between the Duffin-Kemmer-Petiau and the Klein-Gordon and Proca equations," Physical Review A, vol. 90, Article ID 022101, 2014.

[13] L. B. Castro, "Quantum dynamics of scalar bosons in a cosmic string background," The European Physical Journal C, vol. 75, article 287, 2015.

[14] H. S. Snyder, "Quantized space-time," Physical Review, vol. 71, no. 1, pp. 38-41, 1947.

[15] A. Connes, Noncommutative Geometry, Academic Press, San Diego, Calif, USA, 1994.

[16] T. Banks, W. Fischler, S. H. Shenker, and L. Susskind, " $M$ theory as a matrix model: a conjecture," Physical Review D, vol. 55, no. 8, pp. 5112-5128, 1997.

[17] A. E. F. Djemai, "Noncommutative classical mechanics," International Journal of Theoretical Physics, vol. 43, no. 2, pp. 299-314, 2004.

[18] J. Gamboa, M. Loewe, F. Mendez, and J. C. Rojas, "Noncommutative quantum mechanics," Physical Review D, vol. 64, no. 6, Article ID 067901, 2001.

[19] J. M. Romero, J. A. Santiago, and J. D. Vergara, “Newton's second law in a non-commutative space," Physics Letters. A, vol. 310, no. 1, pp. 9-12, 2003.

[20] M. Daszkiewicz and C. J. Walczyk, "Newton equation for canonical, Lie-algebraic, and quadratic deformation of classical space," Physical Review D, vol. 77, no. 10, Article ID 105008, 2008.

[21] J. Jing, F. H. Liu, and J. F. Chen, "Classical and quantum mechanics in the generalized non-commutative plane," $E P L$, vol. 84, no. 6, Article ID 61001, 2008.

[22] B. Muthukumar and P. Mitra, "Noncommutative oscillators and the commutative limit," Physical Review D, vol. 66, no. 2, Article ID 027701, 3 pages, 2002.

[23] A. Kijanka and P. Kosinski, "Noncommutative isotropic harmonic oscillator," Physical Review D, vol. 70, no. 12, Article ID 127702, 2004.

[24] J. Jing, S. H. Zhao, J. F. Chen, and Z. W. Long, "On the spectra of noncommutative 2D harmonic oscillator," The European Physical Journal C, vol. 54, no. 4, pp. 685-690, 2008.

[25] A. Das, H. Falomir, M. Nieto, J. Gamboa, and F. Méndez, "Aharonov-Bohm effect in a class of noncommutative theories," Physical Review D, vol. 84, no. 4, Article ID 045002, 2011. 
[26] G. Guo, C. Long, Z. Yang, and S. Qin, "DKP oscillator in noncommutative phase space," Canadian Journal of Physics, vol. 87, no. 9, pp. 989-993, 2009.

[27] H. Hassanabadi, Z. Molaee, and S. Zarrinkamar, "DKP oscillator in the presence of magnetic field in $(1+2)$-dimensions for spin-zero and spin-one particles in noncommutative phase space," The European Physical Journal C, vol. 72, article 2217, 2012.

[28] Z.-H. Yang, C.-Y. Long, S.-J. Qin, and Z.-W. Long, "DKP oscillator with spin-0 in three-dimensional noncommutative phase space," International Journal of Theoretical Physics, vol. 49, no. 3, pp. 644-651, 2010.

[29] R. Casana, V. Y. Fainberg, B. M. Pimentel, and J. S. Valverde, "Bose-Einstein condensation and free DKP field," Physics Letters A, vol. 316, no. 1-2, pp. 33-43, 2003.

[30] M. H. Pacheco, R. R. Landim, and C. A. S. Almeida, "Onedimensional Dirac oscillator in a thermal bath," Physics Letters, Section A: General, Atomic and Solid State Physics, vol. 311, no. 2-3, pp. 93-96, 2003.

[31] M. H. Pacheco, R. V. Maluf, C. A. S. Almeida, and R. R. Landim, "Three-dimensional Dirac oscillator in a thermal bath," EPL, vol. 108, no. 1, Article ID 10005, 2014.

[32] A. Boumali and H. Hassanabadi, "The thermal properties of a two-dimensional Dirac oscillator under an external magnetic field," The European Physical Journal Plus, vol. 128, article 124, 2013.

[33] H. Hassanabadi, S. S. Hosseini, A. Boumali, and S. Zarrinkamar, "The statistical properties of Klein-Gordon oscillator in noncommutative space," Journal of Mathematical Physics, vol. 55, no. 3, Article ID 033502, 2014.

[34] S. Hassanabadi and M. Ghominejad, "The statistical properties of spin-one DKP oscillator under an external magnetic field in noncommutative space," Advances in High Energy Physics, vol. 2014, Article ID 185169, 7 pages, 2014.

[35] Y. Nedjadi and R. C. Barrett, "The Duffin-Kemmer-Petiau oscillator," Journal of Physics A: Mathematical and General, vol. 27, no. 12, pp. 4301-4315, 1994.

[36] Y. Nedjadi and R. C. Barrett, "On the properties of the DuffinKemmer-Petiau equation," Journal of Physics G: Nuclear and Particle Physics, vol. 19, no. 1, pp. 87-98, 1993.

[37] G. E. Andrews, R. Askey, and A. Roy, Special Functions, Cambridge University Press, Cambridge, UK, 1999.

[38] A. Boumali, "The one-dimensional thermal properties for the relativistic harmonic oscillators," Electronic Journal of Theoretical Physics, vol. 12, no. 32, pp. 121-130, 2015.

[39] A. Boumali, "Thermal properties of the one-dimensional Duffin-Kemmer-Petiau oscillator using Hurwitz zeta function," Zeitschrift für Naturforschung A, vol. 70, no. 10, pp. 867-874, 2015.

[40] A. J. Silenko, "Quantum-mechanical description of spin-1 particles with electric dipole moments," Physical Review D, vol. 87, no. 7, Article ID 073015, 2013.

[41] A. J. Silenko, "High precision description and new properties of a spin-1 particle in a magnetic field," Physical Review D, vol. 89, no. 12, Article ID 121701, 2014.

[42] H. B. Nielsen and M. Ninomiya, "Dirac sea for bosons. 1formulation of negative energy sea for bosons," Progress of Theoretical Physics, vol. 113, no. 3, pp. 603-624, 2005.

[43] H. B. Nielsen and M. Ninomiya, "Dirac Sea for Bosons. IIstudy of the naive vacuum theory for the toy model world prior to filling the negative energy sea," Progress of Theoretical Physics, vol. 113, no. 3, pp. 625-643, 2005.
[44] M.-A. Dariescu and C. Dariescu, "Persistent currents and critical magnetic field in planar dynamics of charged bosons," Journal of Physics Condensed Matter, vol. 19, no. 25, Article ID 256203, 2007. 

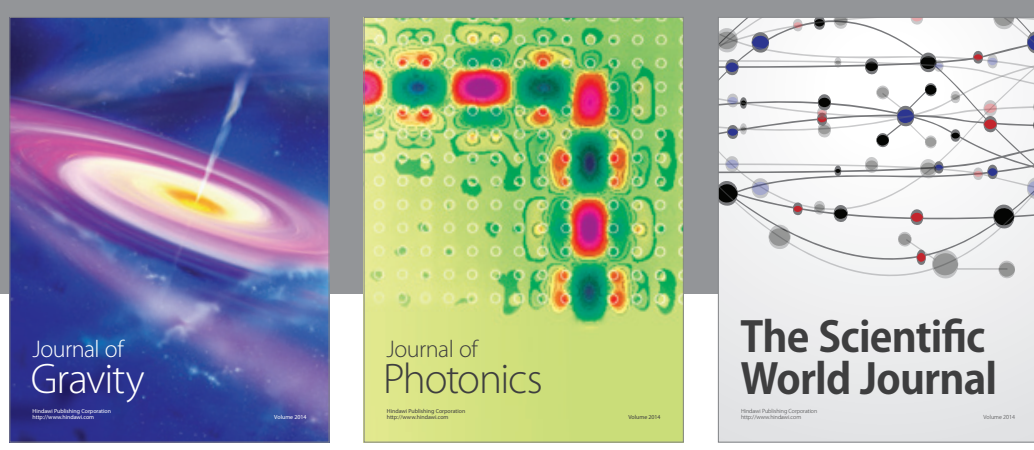

The Scientific World Journal
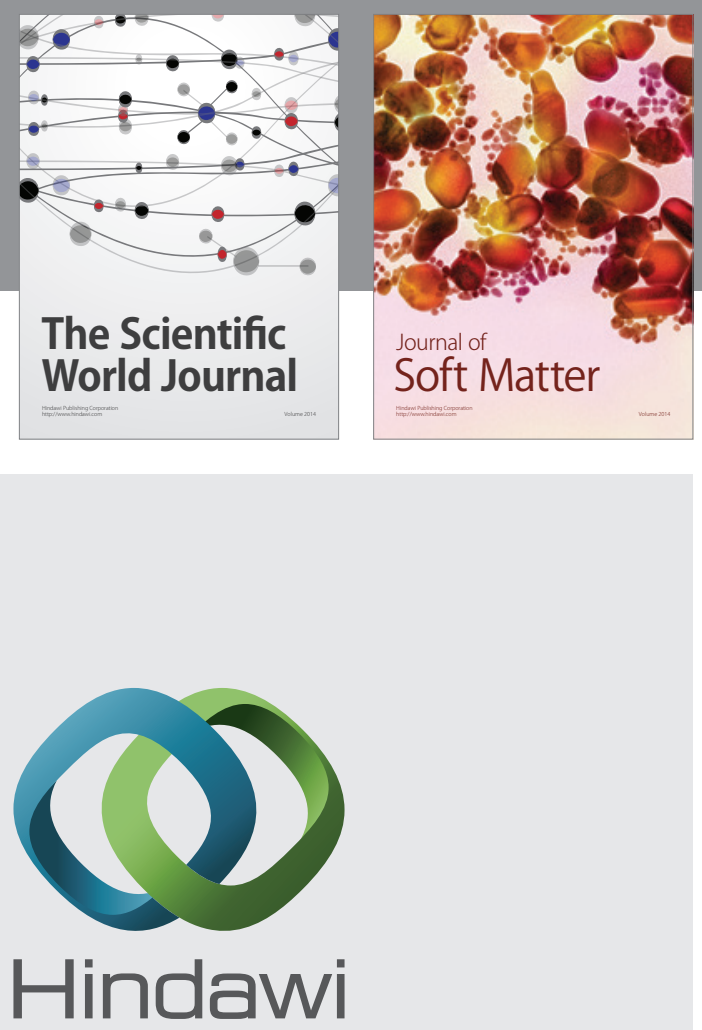

Submit your manuscripts at

http://www.hindawi.com

nternational Journal of

Statistical Mechanics
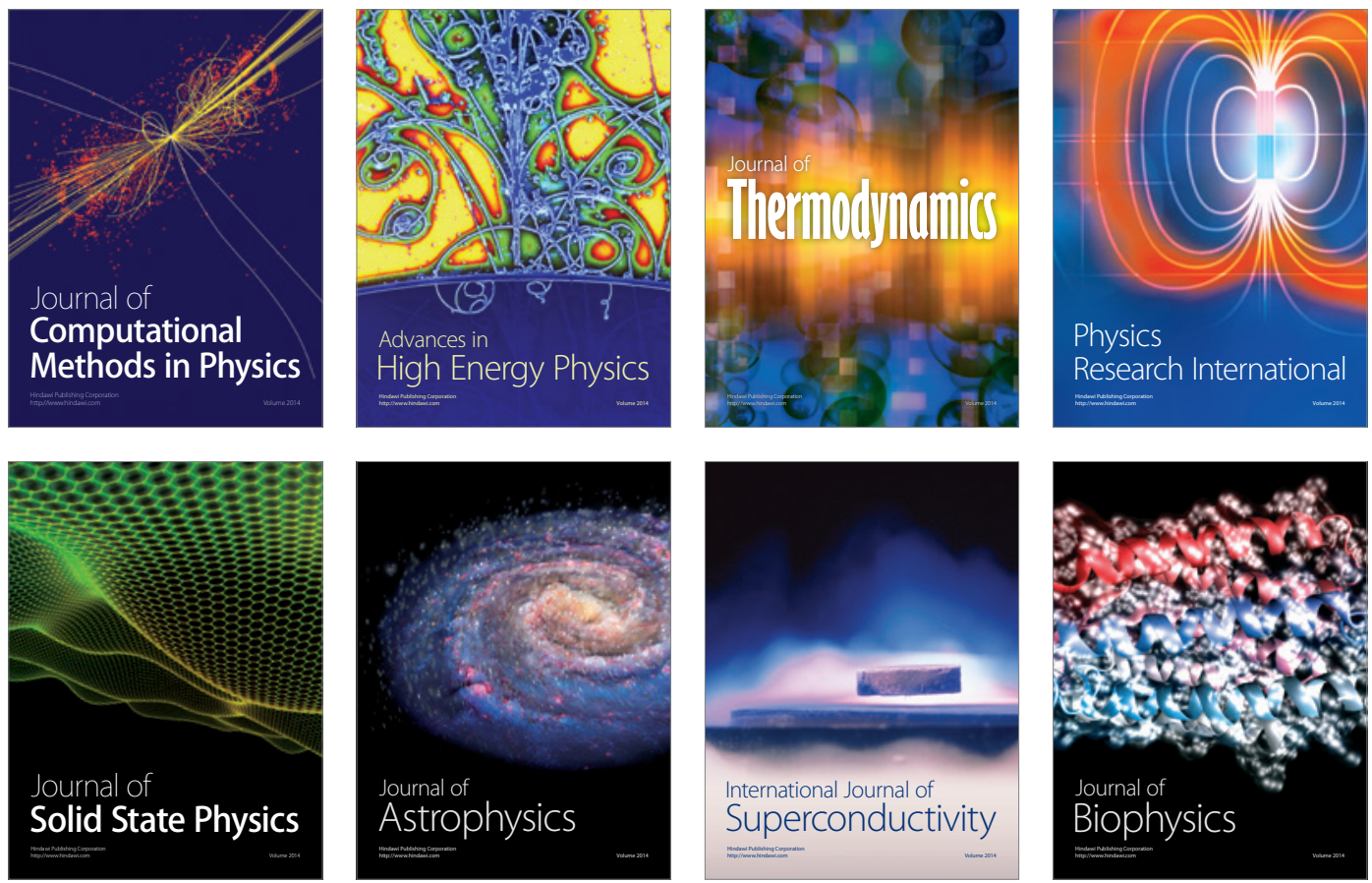
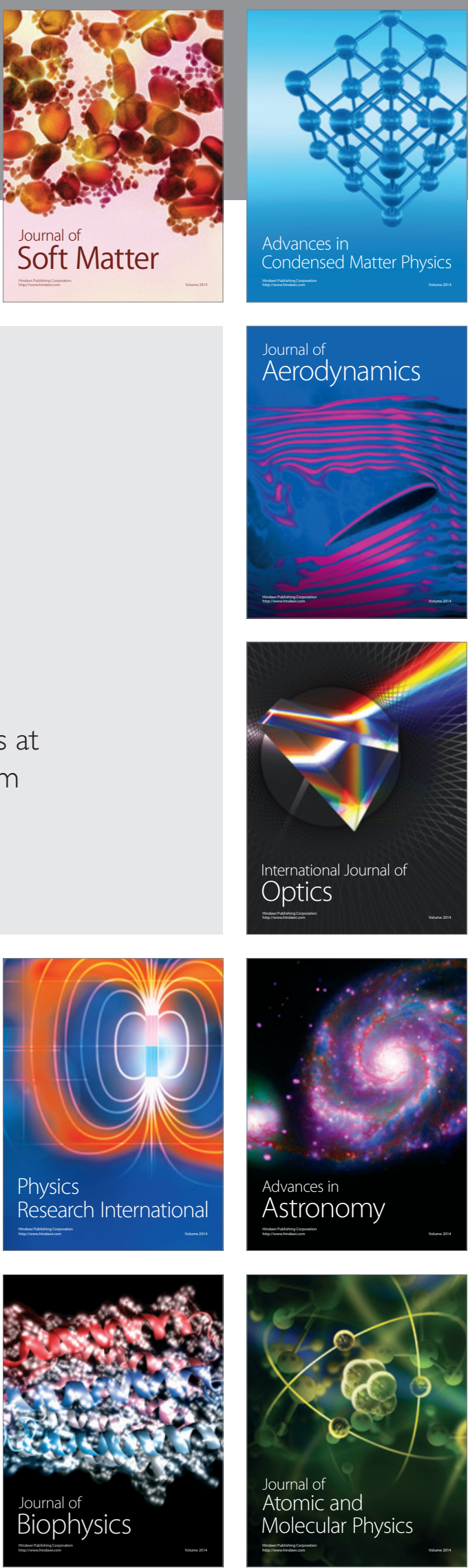